Çukurova Üniversitesi Mühendislik Mimarlık Fakültesi Dergisi, 31(2), ss. 167-177, Aralık 2016

\title{
Jeotermal Rezervuar Modelleme ve JMP Programı ile Model Parametre Değerleri Tahmini
}

\author{
Özen KILIÇ ${ }^{1}$, Sedat TORAMAN ${ }^{* 2}$ \\ ${ }^{I}$ Çukurova Üniversitesi, Mühendislik Mimarlık Fakültesi, Maden Mühendisliği Bölümü, Adana \\ ${ }^{2}$ MTA Orta Anadolu III. Bölge Müdürlüğ̈̈, Ankara
}

Geliş tarihi: 02.06.2016 Kabul tarihi: 23.11.2016

$\ddot{\mathbf{O z}}$

Jeotermal sahaların doğru işletilmesi sahaların doğru modellenmesi ile mümkündür. Jeotermal sahaların modellemesinde, tank modellemesi veri sayısının az olduğu üretimin ilk aşamalarında sıkça kullanılan bir yöntemdir. Ayrıca, tank modellerinin diğer bir avantajı da tarihsel çakıştırmada daha kısa sürede sonuca ulaşılmasıdır. Bu nedenlerle, tank modeller rezervuar özelliklerinde heterojenliği, karmaşık akışkan özelliklerini ve karmaşık kuyu geometrileri gibi zor durumların olmadığı rezervuarların modellemesinde kullanılır. Her bir tank için kütle ve enerji korunum denklemleri yazılarak bu denklemlerin çözülmesiyle ileriye yönelik sıcaklık ve basınç tahminleri yapılabilir. Bu çalışmada, izotermal olmayan tek tank modellemesi kullanılarak Kütahya-Simav jeotermal sahasının modellemesi yapılmıştır. Sahanın model parametreleri interference testi verileri kullanılarak tespit edilmiştir. Model parametre değerleri kalibrasyonu JMP istatistiki programı ile gerçekleştirilmiştir. Modellemede bilgisayar ortamında bir arayüz oluşturulmuş ve bu arayüz üzerinden saha modeli oluşturulmuştur. Model parametre kalibrasyonu ve belirsizlikler tanımlanarak model revize edilmiştir.

Anahtar Kelimeler: Kütahya Simav, Jeotermal saha, Tank modelleme, Model parametreleri tahmini, JMP istatistik programı

\section{Geothermal Reservoir Modeling and Estimation of Model Parameter Values with JMP Program}

\begin{abstract}
In geothermal reservoir modeling, tank modeling is mainly used at the early life of the field when relatively little data is available. Also, the other advantage of tank modeling is that the result is obtained in shorter time during history matching. Because of that reason, tank modeling is used in reservoir which does not have heterogeneous, the fluid in reservoir is not complex and the well doesn't consist of different geometry structure. Mass and energy balance equations are solved on the tanks for making future
\end{abstract}

\footnotetext{
* Sorumlu yazar (Corresponding author): Sedat TORAMAN, sedat.toraman@mta.gov.tr
} 
performance predictions of pressure and temperature. If production data is available, model parameters that best describe the system could be obtained through history matching. In this study, advantages of tank modeling was considered and we have also pointed out a methodology for determining the best model that represents the system. For this purpose we perform history matching with various models and select the one that matches best the production data and the model that gives the lowest confidence intervals for the model parameters. Effect of temperature is neglected at low temperature geothermal reservoir. Although it is important to make accurate predictions of pressure and temperature. In this study we have used the non-isothermal single tank modeling for performing Kütahya-Simav geothermal field modeling. The scope of the model parameters are determined by using interference test data. Calibration of model parameter values are realized with JMP statistics program. An interface has been created on computer and field model has been developed using this interface. Model has been revised by defining calibration of the model parameters and uncertainties.

Keywords: Kütahya Simav, Geothermal field, Tank modeling, Estimate model parameter, Non-lineer regresyon, JMP statistic program

\section{GíRiș}

Jeotermal rezervuar modelleme literatürde genel olarak sayısal ya da tank modelleri olmak üzere iki şekilde yapılmaktadır. Sayısal modellerin çalışma prensibi herhangi bir rezervuarı hücrelere bölerek her bir hücre üstünde kütle dengesi ve enerji dengesi denklemlerinin çözülmesine dayanır. Tank modellerinde ise jeotermal sistemin içindeki rezervuar ve akifer tanklar tarafindan temsil edilmektedir. Kütle ve enerji dengesi denklemlerinin tanklar üstünde çözülmesiyle rezervuar ve akiferlerin ortalama basınç ve sıcaklık davranışları modellenebilir. Sayısal modellerin karmaşıklı̆g 1 ve detaylı saha verilerine ihtiyaç duymaları nedeniyle tank modelleme daha fazla tercih edilmektedir. Özellikle ülkemizdeki birçok sahanın yeni işletmeye alınmaları nedeniyle sayısal modeller için ihtiyaç duyulan detaylı veriler olmadığından sahaların tank modelleriyle modellenmesi tercih sebebi olmaktadır.

Jeotermal rezervuar modelleme çalışmaları kütle korunum denklemi ve enerji korunum denklemlerinin birlikte çözümlemesi ile gerçekleştirilmekte olup bu denklem çözümlerinde ihtiyaç duyulan ve rezervuar karakteristik özelliklerini içeren parametrelerin doğru tespiti yapılacak modellemenin de doğru sonuçlar vermesi için önemlidir. Bu kapsamda jeotermal sahalarda yapılan kuyu testleri çok önemlidir. Kuyu test çalışmaları değerlendirilerek birçok saha karakteristik özelliklerini içeren parametrelerin tahmini yapilabilmektedir. Model parametre değerlerinin tahmini doğrusal olmayan regresyon analizi ile de mümkündür. Bir çok istatistik programı non-lineer fit modül ile parametre tahmini yapmakta ve belirsizlikleri ortaya koymaktadır.

$\mathrm{Bu}$ çalı̧̧mada, izotermal olmayan tek tank modellemesi kullanılarak Kütahya-Simav jeotermal sahasının JMP Programı ile model parametre değerleri tahmini yapılmıştır.

\section{JEOTERMAL REZERVUARLAR IÇIIN TANK MODELLEME}

Whiting ve Ramey [1] lumped parametre modeli kullanarak rezervuar özelliklerini belirlemeye çalışmıştır. Brigham ve Morrow [2] lumped parametre modelini buhar baskın bir sistemde uygulamıştır. Bodvarsson ve Pruess [3], Krafla jeotermal alanında rezervuarın gelecekteki performansının tahmini için dağınık parametreli model çalışması yapmıştır. Bodvarsson ve arkadaşları [4], jeotermal rezervuarların modellenmesine yönelik teorik bir çalışma gerçekleştirmiştir. Bu çalışma kapsamında farklı modelleme yaklaşımlarını tanımlamış, bunların avantajını ve limitlerini tartışmışlardır. Kütle ve 1s1 taşınım eşitliklerinin oluşturulması ve bunların çözüm teknikleri ile jeotermal rezervuarların modellemesinde karşılaşılan problemleri ortaya koymuşlardır. Alkan ve Satman [5] boyutsuz 
parametre modelini karbondioksit içeren jeotermal rezervuarlar için geliştirmiştir. Sarak [6] düşük sıcaklıklı jeotermal rezervuarlar için çeşitli konfigürasyonlardaki tank modeller için analitik denklemler geliştirmiştir. Onur ve arkadaşları [7] izotermal olmayan boyutsuz parametre modelini sıvı ağırlıklı düşük sıcaklıklı jeotermal rezervuarlar için geliştirmiştir. $\mathrm{Bu}$ çalışmanın bir adım ilerisi olarak Türeyen ve arkadaşları [8] izotermal olmayan sıvı ağırlıklı jeotermal rezervuarlar için boyutsuz parametre modelini geliştirmişlerdir.

Tank modellemesi parametrelerin ve verilerin az olduğu durumlarda sayısal modellere alternatif olarak kullanılan bir yöntemdir. Rezervuar üretim programını belirlemek ve üretim performansını artırmak için kullanılır. Tank modellerinde rezervuar ve akifer homojen tanklar olarak tanımlanır. Tank modelleri rezervuar, akifer ve besleme kaynağı olmak üzere üç ana bölümden oluşmaktadır. Bunlardan rezervuar ve akifer ortalama özelliklere sahip homojen tanklar tarafından temsil edilmektedir. Besleme kaynağ 1 , bu tanklardan herhangi birine (akifer ya da rezervuarın kendisine) ya da tüm tanklara bağlanabilmekte ve sistemin sabit basınçlı dış sınırını temsil etmektedir. Eğer tank modeli beslenme kaynağına bağlı ise açık sistem, bağlı değil ise kapalı sistem olarak adlandırılır. Tank modellerinde basınç ve sıcaklık hesaplamalarının temelini kütlenin ve enerjinin korunumu denklemlerinin tank için çözülmesi oluşturur. Tank modelleri izotermal ve non-izotermal tank modelleri olmak üzere iki gruba ayrılır.

\section{1. İzotermal Tank Modelleri}

Jeotermal sistemi temsil eden tanklar üstünde sadece kütlenin korunumu denklemleri çözülürse, bu modellere izotermal modeller denir.

\subsection{Non-İzotermal Tank Modelleri}

Kütle dengesi denklemiyle birlikte enerji dengesi denklemlerinin çözülmesiyle izotermal olmayan modeller elde edilir. $\mathrm{Bu}$ modeller sayesinde jeotermal sistemdeki akiferlerin ve rezervuarın basınç davranışlarıyla birlikte sıcaklık davranışları da modellenebilmektedir. Şekil 1'de genelleştirilmiş izotermal olmayan tank modeli görülmektedir. Tank modellemelerde kullanılan kütle ve 1 sı taşınım denklemleri Eşitlik 1'de verilmiştir.

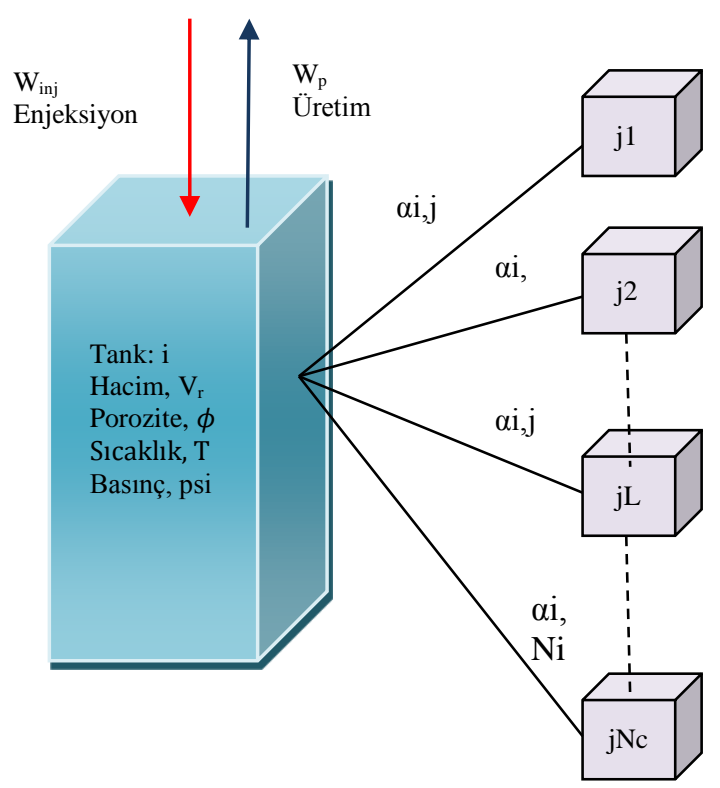

Şekil 1. Genelleştirilmiş izotermal olmayan model

$\mathrm{V}_{\mathrm{r}} \frac{\mathrm{d}\left(\rho_{\mathrm{w}} \phi_{\mathrm{r}}\right)}{\mathrm{dt}}+\left[\mathrm{W}_{\mathrm{p}}(\mathrm{t})-\mathrm{W}_{\text {inj }}(\mathrm{t})\right]-\alpha_{\mathrm{s}}\left[\mathrm{p}_{\mathrm{i}}-\mathrm{p}(\mathrm{t})\right]=0$

Eşitlik 1'de verilen kütle korunum denklemindeki ilk terim rezervuarda biriken kütle debisini, ikinci terim net üretim debisini ve son terim ise bağlı olan tankların net akış debisini ifade eder. Isı taşınım denklemi Eşitlik 2'de verilmiştir. Eşitlik 2'de ilk terim akışkanda ve kaya içinde biriken enerjiyi, ikinci terim enjekte edilen akışkandan sisteme giren enerjiyi ve üçüncü terim üretim nedeniyle sistemden çekilen enerjiyi ve son olarak dördüncü terim ise bağlı olan tanklardan sisteme giren enerjiyi ifade eder.

Saha modellemede asıl olan, sahanın karakteristik özelliklerini yansıtan parametrelerin doğru tespitidir. Sahada yapılan jeolojik, jeofizik, sondaj ve kuyu testlerinin birlikte değerlendirilmesi ile doğru parametre değerleri saptanabilir. Doğru saptanamayan parametreler, modelleme çalışmasında tekrar tekrar başa dönülmesine sebep olmaktadır. Bu nedenle, modelleme çalışmasına başlamadan önce saha verileri özenle tetkik edilmelidir. 
$\frac{d}{d t}\left[\left(1-\phi_{r}\right) V_{r} \rho_{m} C_{m} T+V_{r} \phi_{r} \rho_{w} U_{w}\right]-W_{i n j}(t) h_{w, i n j}(t)+W_{p}(t) h_{w, p}(t)-\alpha_{s}\left[p_{i}-p(t)\right] h_{w, s}(t)=0$

Burada;

$\mathrm{V}_{\mathrm{r}} \quad$ : Rezervuar kaba hacmi $\left(\mathrm{m}^{3}\right)$

$\rho_{\mathrm{m}} \quad$ : Kayaç katı kısmı yoğunluğu, $\mathrm{kg} / \mathrm{m}^{3}$

$\mathrm{C}_{\mathrm{m}} \quad$ : Kayaç katı kısmı özgül 1sı kap., $\mathrm{KJ} /\left(\mathrm{kg}{ }^{\circ} \mathrm{C}\right)$

$\mathrm{T} \quad$ : Rezervuar ortalama sicaklığ $1,{ }^{\circ} \mathrm{C}$

$\phi_{\mathrm{r}} \quad$ : Rezervuar gözeneklilik değeri

$\rho_{\mathrm{w}} \quad$ : Jeotermal suyun yoğunluğu, $\mathrm{kg} / \mathrm{m}^{3}$

$\mathrm{U}_{\mathrm{w}} \quad$ : Jeotermal suyun özgül içsel enerjisi, $\mathrm{KJ} / \mathrm{kg}$

$\mathrm{h}_{\mathrm{w}, \mathrm{s}}:$ :Beslenme suyu özgül entalpisi, $\mathrm{KJ} / \mathrm{kg}$

$\mathrm{h}_{\mathrm{w}, \text { inj }}$ : Enjeksiyon suyun özgül entalpisi, KJ/kg

$\mathrm{h}_{\mathrm{w}, \mathrm{p}}$ : Üretilen suyun özgül entalpisi, $\mathrm{KJ} / \mathrm{kg}$

$\alpha_{s} \quad$ : Beslenme miktarı kg/s

$\mathrm{W}_{\mathrm{p}}:$ Üretim miktarı $\mathrm{kg} / \mathrm{s}$

$\mathrm{W}_{\text {inj }}$ : Enjeksiyon miktarı $\mathrm{kg} / \mathrm{s}$

İzotermal olmayan modelleme çalışmasında 1S1 korunum ve kütle korunum denklemleri birlikte çözümlendiğinden, her iki denklemde yer alan parametrelerin değerleri saptanmalıdır (Eşitlik 3-5).

1- Kütle korunum,
İzotermal olmayan model denklemleri ve porozitenin basınç-sıcaklıkla değişim denklemi incelendiğinde model denklem parametreleri üç grupta toplanabilir. Bunlar; rezervuar kayaç özellikleri, akışkan karakteristik özellikleri ve saha üretim/beslenme ve enjeksiyon bilgileridir.

Kütle ve enerji denklemleri doğrusal olmayan diferansiyel denklemlerdir. $\mathrm{Bu}$ nedenle, tümüyle kapalı Newton-Raphson yineleme yöntemiyle [9] birlikte çözümlemek gerekmektedir. Çözümlemede birincil değişkenler olarak basınç (p) ve sıcaklık (T) göz önünde bulundurulmaktadır. Denklemler bu değişkenler için zamanın fonksiyonu olarak bir algoritma ile çözümlenmektedir. Kütle denklemi ve enerji denklemi herhangi bir $\mathrm{t}^{\mathrm{n}+1}$ zamanında $(\mathrm{n}=0,1,2, \ldots) \quad R_{i}\left(p^{n+1}, T^{n+1}\right)=0 \quad \mathrm{i}=1 \quad$ (kütle denklemi), $\mathrm{i}=2$ (enerji denklemi) ile tümüyle kapalı olarak ifade edilmektedir. Newton-Raphson yönteminin $\mathrm{k}$. yineleme adımındaki basınç ve sıcaklığı ihtiva eden vektör, $\mathrm{w}^{\mathrm{n}+1, \mathrm{k}}$ Eşitlik 6'daki gibi tanımlanmaktadır.

$R_{1}\left(p^{n+1}, T^{n+1}\right)=\left(\rho_{w} \phi_{r}\right)^{n+1}-\left(\rho_{w} \phi_{r}\right)^{n}-\frac{\alpha_{s} \Delta t_{n+1}}{V_{r}}\left[p_{i}-p(t)\right]+\frac{\Delta t_{n+1}}{V_{r}}\left(W_{p}^{n+1}-W_{i n j}^{n+1}\right)=0$

2- Isı taşınım denklemi,

$$
\begin{aligned}
R_{2}\left(p^{n+1}, T^{n+1}\right) & \\
= & \left(1-\phi_{i}\right) \rho_{m} C_{m}\left(T^{n+1}-T^{n}\right)+\left[\phi_{r}^{n+1} \rho_{w}^{n+1} U_{w}^{n+1}-\phi_{r}^{n} \rho_{w} U_{w}\right] \\
& -\frac{\Delta t_{n+1}}{V_{r}} W_{i n j}^{n+1} h_{w_{i} i n j}-\alpha_{s} \frac{\Delta t_{n+1}}{V_{r}}\left[p_{i}-p^{n+1}\right] h_{w, s}+\frac{\Delta t_{n+1}}{V_{r}} W_{p}^{n+1} h_{w, p}^{n+1}
\end{aligned}
$$

3- Porozitenin basınç ve sıcaklıkla değişimi,

$\phi(p, T)=\phi_{i}\left[1+c_{r}\left(p(t)-p_{0}\right)-\beta_{r}\left(T(t)-T_{0}\right]\right.$

$\mathrm{W}^{\mathrm{n}+1, \mathrm{k}}=\left[\begin{array}{c}\mathrm{W}_{1}^{\mathrm{n}+1, \mathrm{k}} \\ \mathrm{W}_{2}^{\mathrm{n}+1, \mathrm{k}}\end{array}\right]=\left[\begin{array}{c}\mathrm{p}^{\mathrm{n}+1, \mathrm{k}} \\ \mathrm{T}^{\mathrm{n}+1, \mathrm{k}}\end{array}\right]$

$\mathrm{J}^{\mathrm{n}+1, \mathrm{k}} \delta \mathrm{w}^{\mathrm{n}+1, \mathrm{k}+1}=-\mathrm{R}^{\mathrm{n}+1, \mathrm{k}}$

Verilen bir ilk tahmin $\mathrm{w}^{\mathrm{n}+1,0}, \mathrm{k}=0,1,2, \ldots$ olacak şekilde matris vektör denklemi Newton-Raphson yöntemiyle çözümlenmektedir (Eşitlik 7).

Burada $\mathrm{J}$ bir önceki yineleme adımındaki çözüm vektörü $\mathrm{w}^{\mathrm{n}+1, \mathrm{k}}$ değerinde hesaplanmış "Jacobian" matrisini temsil etmektedir (Eşitlik 8). 
$\mathbf{J}^{\mathrm{n}+1, \mathrm{k}}=\left[\begin{array}{cc}\frac{\partial \mathrm{R}_{1}}{\partial \mathrm{p}^{\mathrm{n}+1}} & \frac{\partial \mathrm{R}_{1}}{\partial \mathrm{T}^{\mathrm{n}+1}} \\ \frac{\partial \mathrm{R}_{2}}{\partial \mathrm{p}^{\mathrm{n}+1}} & \frac{\partial \mathrm{R}_{2}}{\partial \mathrm{T}^{\mathrm{n}+1}}\end{array}\right] \quad \mathrm{R}^{\mathrm{n}+1, \mathrm{k}}=\left[\begin{array}{c}\mathrm{R}_{1} \\ \mathrm{R}_{2}\end{array}\right] \quad$ ise $\quad\left|\begin{array}{cc}\frac{\partial \mathrm{R}_{1}}{\partial \mathrm{p}^{\mathrm{n}+1}} & \frac{\partial \mathrm{R}_{1}}{\partial \mathrm{T}^{\mathrm{n}+1}} \\ \frac{\partial \mathrm{R}_{2}}{\partial \mathrm{p}^{\mathrm{n}+1}} & \frac{\partial \mathrm{R}_{2}}{\partial \mathrm{T}^{\mathrm{n}+1}}\end{array}\right|\left|\begin{array}{c}\Delta \mathrm{P}^{\mathrm{n}+1} \\ \Delta \mathrm{T}^{\mathrm{n}+1}\end{array}\right|=-\left|\begin{array}{c}\mathrm{R}_{1} \\ \mathrm{R}_{2}\end{array}\right|$

$\mathrm{P}^{\mathrm{n}+1}$ ve $\mathrm{T}^{\mathrm{n}+1}$ değerlerinin hesaplanmasında kullanılan basınç ve sıcaklık değişimleri Eşitlik 9 ve Eşitlik 10'da verilmiştir:

$$
\begin{aligned}
& \Delta \mathrm{P}=\frac{-\mathrm{R}_{2} * \frac{\partial \mathrm{R}_{1}}{\partial \mathrm{T}^{\mathrm{n}+1}}-\mathrm{R}_{1} * \frac{\partial \mathrm{R}_{2}}{\partial \mathrm{T}^{\mathrm{n}+1}}}{\frac{\partial \mathrm{R}_{1}}{\partial \mathrm{T}^{\mathrm{n}+1}} * \frac{\partial \mathrm{R}_{2}}{\partial \mathrm{p}^{\mathrm{n}+1}}+\frac{\partial \mathrm{R}_{1}}{\partial \mathrm{p}^{\mathrm{n}+1}} * \frac{\partial \mathrm{R}_{2}}{\partial \mathrm{T}^{\mathrm{n}+1}}} \\
& \Delta \mathrm{T}=\frac{-\frac{\partial \mathrm{R}_{1}}{\partial \mathrm{p}^{\mathrm{n}+1}} * \Delta \mathrm{P}+\mathrm{R}_{1}}{\frac{\partial \mathrm{R}_{1}}{\partial \mathrm{T}^{\mathrm{n}+1}}}
\end{aligned}
$$

\subsection{Tarihsel Çakıştırma ve Doğrusal Olmayan Parametre Tahmini}

Model parametrelerinin belirlenmesinde, rezervuarların üretim/re-enjeksiyon esnasında elde edilen veriler kullanılır. Belirli bir süre üretim yapmış rezervuardan gelen veriler, tank modellerinden alınan veriler ile çakıştırma yapılarak rezervuar parametreleri tayin edilir. Başka bir deyişle, ilk olarak rezervuar parametreleri tahmin edilir ve tank modellerinden elde edilen basınç veya su seviyesi değerleri sahadan gelen verilerle uyum sağlayıncaya kadar değiştirilir. Model parametreleri belirlenen rezervuarların geleceğe yönelik davranışlarının tahmininde de aynı yöntem uygulanır. Tahmin edilen parametreler kullanılarak tank modelleri ile geleceğe yönelik basınç tahminleri yapılır.

Bilgisayar destekli doğrusal olmayan eğri çakıştırma yöntemleri ile çok kısa süreler içerisinde ve etkin bir şekilde tarihsel çakıştırma yapılabilmektedir. Tarihsel çakıştırmanın temel nedeni, model ve ölçüm verilerindeki hataların (belirsizliklerin) hesaba katılması ve model ile ölçüm çakışmasından elde edilen parametrelerin istatistiksel olarak nicelik bakımından değerlendirilmesinin mümkün olmasıdır. $\mathrm{Bu}$ avantajları nedeniyle bilgisayar destekli doğrusal olmayan eğri çakıştırma yöntemleri, dinamik verilere çakıştırma işlemi ile model parametrelerin belirlenmesinde temel bir araç haline gelmiştir.
Doğrusal olmayan parametre tahmin yöntemlerinde, geçmiş dinamik verilere çakıştırma işlemi, doğrusal olmayan optimizasyon problemi olarak formüle edilir ve genelde çakıştırma ağırlıklı en küçük kareler yöntemi ile yapılır. Ağrılıklı en küçük kareler yönteminde bilinmeyen model parametre vektörü $\mathrm{J}(\bar{\chi})^{\prime}$ 'e göre minimize edilecek hedef fonksiyon Eşitlik 11'de verildiği şekliyle tanımlanabilir.

$\mathrm{J}(\vec{\chi})=\sum_{\mathrm{j}=1}^{\mathrm{M}} \sum_{\mathrm{i}=1}^{\mathrm{nj}} \mathrm{W}_{\mathrm{j}, \mathrm{i}}\left[\mathrm{f}_{\mathrm{j}}\left(\mathrm{t}_{\mathrm{i}}, \vec{\chi}\right)-\mathrm{y}_{\mathrm{j}}\left(\mathrm{t}_{\mathrm{i}}\right)\right]^{2}$

M, toplam model fonksiyonu (f) sayısını, (ti, yj(ti) $\mathrm{i}=1 \ldots$...nj model fonksiyonu $\mathrm{fj}(\mathrm{j}=1 \ldots, \mathrm{M})$ için yapılmış nj gözlemden oluşan veri setini temsil etmektedir. $\vec{\chi}, \quad$ göz önünde bulundurulan modeldeki bilinmeyen model parametrelerini içeren, bilinmeyen model parametre vektörüdür. Eşitlik 11'de wj,i, her ölçüm verisi için pozitif ağırlıkları temsil eder. $\mathrm{Bu}$ ağırlıklar, herhangi bir zaman değeri $t_{i}$ 'de ölçülen $y_{j}$ için, ölçüm hatalarının varyansının (saçılım değerinin) tersini temsil eder. Eğer verilen bir ölçüm için ağırlık sıfıra yakın (veya başka deyişle ölçüm noktası için varyans, belirsizlik, büyük) ise, o ölçüm verisi güvenilir değildir ve çakıştırmada o veri noktasına daha az ağırlık atanması mümkün olur. Uygulamalarda, yj'ler rezervuar basınc1 ölçümlerini temsil eder. Ancak, Eşitlik 11 ile tanımlanan hedef fonksiyon, birden fazla ve farklı veri setini (örneğin izotermal olmayan ve boyutlu modellerde) farklı kuyularda ölçülmüş sıcaklık, basınç, izleyici madde konsantrasyonlarını aynı anda çakıștırmak için kullanılacak kadar da geneldir. Eşitlik 11'in minimizasyonu, farklı yöntemlerle yapılabilmektedir. Yaygın olarak kullanılanı gradyant temelli Levenberg-Marquardt yöntemidir. Bu yöntemde yineleme işlemi ve yakınsama kıstasları kullanılarak, ölçüm verilerine en iyi çakışma sağlanarak, "en iyi" (optimum) model parametre vektörü $\bar{\chi}$ tahmin edilmektedir. Doğrusal olmayan parametre tahmin yöntemlerinde, geçmiş dinamik verilere çakıştırma 
işlemi, doğrusal olmayan optimizasyon problemi olarak formüle edilir ve bunun için çeşitli istatistiki hazır programlar mevcuttur [8]. Non-linear parametre tahmini algoritması Eşitlik $12-15$ 'de verilen formüllere göre yapılmaktadır.

Ölçüm $\mathrm{P}=\operatorname{Model} \mathrm{P}\left(\Theta_{1}, \Theta_{2}, \Theta_{3 \ldots \ldots . .} \Theta_{\mathrm{n}}\right)_{+\epsilon} y_{i}=f\left(x_{i}\right)+€$

$\left(\mathrm{x}_{\mathrm{i}}\right)_{\mathrm{j}+1}=\mathrm{f}\left(\mathrm{x}_{\mathrm{i}}\right)_{\mathrm{j}}+\frac{\partial \mathrm{f}\left(\mathrm{x}_{\mathrm{i}}\right)_{\mathrm{j}}}{\partial \Theta_{1}} \Delta \Theta_{1}+\frac{\partial \mathrm{f}\left(\mathrm{x}_{\mathrm{i}}\right)_{\mathrm{j}}}{\partial \Theta_{2}} \Delta \Theta_{2}+\frac{\partial \mathrm{f}\left(\mathrm{x}_{\mathrm{i}}\right)_{\mathrm{j}}}{\partial \Theta_{3}} \Delta \Theta_{3}+\ldots+\frac{\partial \mathrm{f}\left(\mathrm{x}_{\mathrm{i}}\right)_{\mathrm{j}}}{\partial \Theta_{\mathrm{n}}} \Delta \Theta_{\mathrm{n}}$

$\mathrm{y}_{\mathrm{i}}=\mathrm{f}\left(\mathrm{x}_{\mathrm{i}}\right)_{\mathrm{j}}+\frac{\partial \mathrm{f}\left(\mathrm{x}_{\mathrm{i}}\right)_{\mathrm{j}}}{\partial \Theta_{1}} \Delta \Theta_{1}+\frac{\partial \mathrm{f}\left(\mathrm{x}_{\mathrm{i}}\right)_{\mathrm{j}}}{\partial \Theta_{2}} \Delta \Theta_{2}+\frac{\partial \mathrm{f}\left(\mathrm{x}_{\mathrm{i}}\right)_{\mathrm{j}}}{\partial \Theta_{3}} \Delta \Theta_{3}+\ldots+\frac{\partial \mathrm{f}\left(\mathrm{x}_{\mathrm{i}}\right)_{\mathrm{j}}}{\partial \Theta_{\mathrm{n}}} \Delta \Theta_{\mathrm{n}}+\mathrm{C}$

$\mathrm{y}_{\mathrm{i}}-\mathrm{f}\left(\mathrm{x}_{\mathrm{i}}\right)_{\mathrm{j}}=\frac{\partial \mathrm{f}\left(\mathrm{x}_{\mathrm{i}}\right)_{\mathrm{j}}}{\partial \Theta_{1}} \Delta \Theta_{1}+\frac{\partial \mathrm{f}\left(\mathrm{x}_{\mathrm{i}}\right)_{\mathrm{j}}}{\partial \Theta_{2}} \Delta \Theta_{2}+\frac{\partial \mathrm{f}\left(\mathrm{x}_{\mathrm{i}}\right)_{\mathrm{j}}}{\partial \Theta_{3}} \Delta \Theta_{3}+\ldots+\frac{\partial \mathrm{f}\left(\mathrm{x}_{\mathrm{i}}\right)_{\mathrm{j}}}{\partial \Theta_{\mathrm{n}}} \Delta \Theta_{\mathrm{n}}+\mathrm{C}$

Denklem matris ve vektörlerle ifade edildiğinde; $\{D\}=\left[Z_{j}\right]\{\Delta \Theta\}+[\epsilon]$

Burada;

\begin{tabular}{|c|c|c|}
\hline Kuvvet vektörü & Jacobian matrisi & Çözüm vektörü \\
\hline $\mathrm{D}\}=\left[\begin{array}{c}\mathrm{y}_{\mathrm{i}-} \mathrm{f}\left(\mathrm{x}_{1}\right) \\
\mathrm{y}_{\mathrm{i}-\mathrm{f}} \mathrm{f}\left(\mathrm{x}_{2}\right) \\
\cdot \\
\cdot \\
\cdot \\
\mathrm{y}_{\mathrm{i}-\mathrm{f}\left(\mathrm{x}_{2}\right)}\end{array}\right]$ & {$\left[\mathrm{Z}_{\mathrm{j}}\right]=\left[\begin{array}{ccccc}\frac{\partial \mathrm{f}_{1}}{\partial \Theta_{1}} & \frac{\partial \mathrm{f}_{1}}{\partial \Theta_{2}} & \frac{\partial \mathrm{f}_{1}}{\partial \Theta_{3}} & \cdot & \cdot \frac{\partial \mathrm{f}_{1}}{\partial \Theta_{\mathrm{n}}} \\
\frac{\partial \mathrm{f}_{2}}{\partial \Theta_{1}} & \frac{\partial \mathrm{f}_{2}}{\partial \Theta_{2}} & \frac{\partial \mathrm{f}_{2}}{\partial \Theta_{3}} & \cdot & \frac{\partial \mathrm{f}_{2}}{\partial \Theta_{\mathrm{n}}}\end{array}\right]$} & $\{\Delta \Theta\}=\left[\begin{array}{c}\Delta \Theta_{1} \\
\Delta \Theta_{2} \\
\Delta \Theta_{3} \\
\cdot \\
. \\
\Delta \Theta_{\mathrm{n}}\end{array}\right]$ \\
\hline
\end{tabular}

\section{MATERYAL VE YÖNTEM}

Çalışmada doğrusal olmayan parametre tahmin işlemi SAS INSTITUTE INC.JMP 10.0.0 (demo) istatistiki program yardımı ile yapılmıştır. İzotermal olmayan akış modeli ile ölçüm verileri çakıştırması, hem basınç hem de sıcaklık verilerine birlikte veya yalnızca basınç ve yalnızca sıcaklık verilerine yapılabilmektedir. Ancak, KütahyaSimav sahasinda yapılan interference test çalışmalarında sadece kuyu dibi basınç verileri kaydedildiğinden, çalışmada sadece basınç verilerine çakıştırma gerçekleştirilmiş ve çakıştırma neticesinde tahmin edilen parametre değerleri kullanılarak, sahadaki sıcaklık profil değişimi hesaplanmıştır [10].

Sahada gerçekleştirilen basınç değişim takibi eğer mekanik elementler ile gerçekleștirilmiş ise gözlemlenmiş basınç değerlerine gürültü eklenmelidir. Kütahya-Simav sahasında gerçekleştirilen basınç değişim takibi dijital element yardımıyla yapıldığından ölçüm (gözlemlenmiş) basınç değerlerine gürültü eklenmemiştir (Şekil 2).

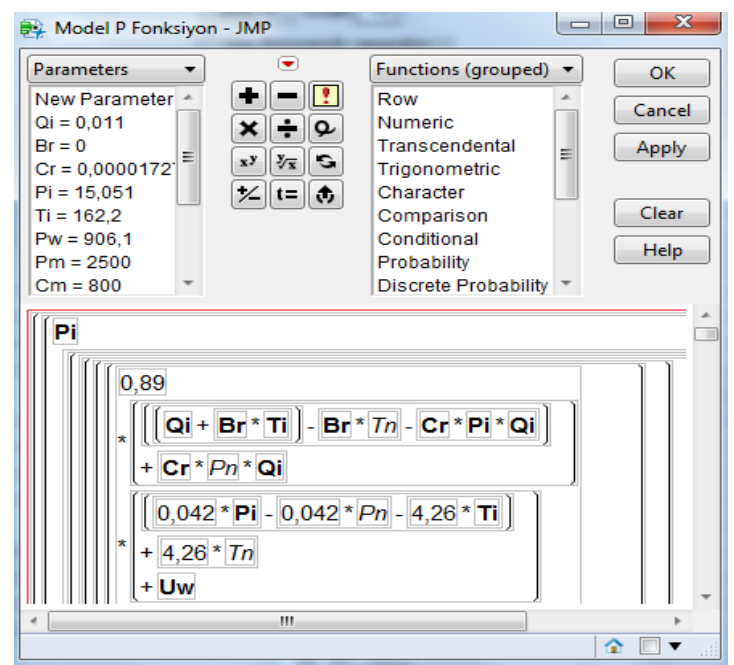

Şekil 2. Model fonksiyon ve parametrelerin programa işlenmesi 
Nonlineer regresyona başlamadan önce model basınç fonksiyonu programa eklenir. Ayrıca tahmin edilecek parametrelerin tanımlamaları ve değerleri sisteme işlenir (Şekil 3).

Model basınç fonksiyonu;

Pmodel $=\mathrm{Pi}-\frac{R_{2} * \frac{\partial R_{1}}{\partial T^{n+1}}-R_{1} * \frac{\partial R_{2}}{\partial T^{n+1}}}{\frac{\partial R_{1}}{\partial T^{n+1}} * \frac{\partial R_{2}}{\partial p^{n+1}}+\frac{\partial R_{1}}{\partial p^{n+1}} * \frac{\partial R_{2}}{\partial T^{n+1}}}+\mathrm{Cnr}$

$\frac{-R_{2} * \frac{\partial R_{1}}{\partial T^{n+1}}-R_{1} * \frac{\partial R_{2}}{\partial T^{n+1}}}{\frac{\partial R_{1}}{\partial T^{n+1}} * \frac{\partial R_{2}}{\partial p^{n+1}}+\frac{\partial R_{1}}{\partial p^{n+1}} * \frac{\partial R_{2}}{\partial T^{n+1}}}=\Delta P^{\prime} \mathrm{yi}^{\prime}$

JMP programı Analyz/modelling sekmesinde non-lineer modül çalıştırıldığında tahmin edilebilecek porozite, kayaç sıkıştırılabilirliği, ilk basınç, ilk sıcaklık, rezervuar kaba hacmi, akışkan yoğunluğu, kayaç yoğunluğu, kayaç ve akışkan özellikleri ile beslenme indeksi de dahil olmak üzere, toplam 14 parametre tanımlanan model değerleriyle modülde gelir (Șekil 4). Modülde regresyon kriterleri istenilen ölçülerde değiştirilebilir durumdadır. Ayrıca, regresyonda tahmin edilecek parametreler dışındaki parametreler analiz setinden çıkarılmak isteniyorsa, "lock" sütununda o parametre kutucuğu işaretlenir. İşaretlenen parametreler regresyonda bilinen parametre olarak kabul edilir. Kütahya-Simav jeotermal sahası için doğrusal olmayan parametre tahmin ișlemi 357. iterasyonda sonuca ulaşılmıştır (Şekil 5). Regresyon sonucu çözüm değerleri ve parametreler arası korelasyon Şekil 6'daki gibi çıkmıştır.

\section{Pi: İlk Basınç değeri}

Enr: Newton-Raphson regrasyon analiz artığını ifade eder.

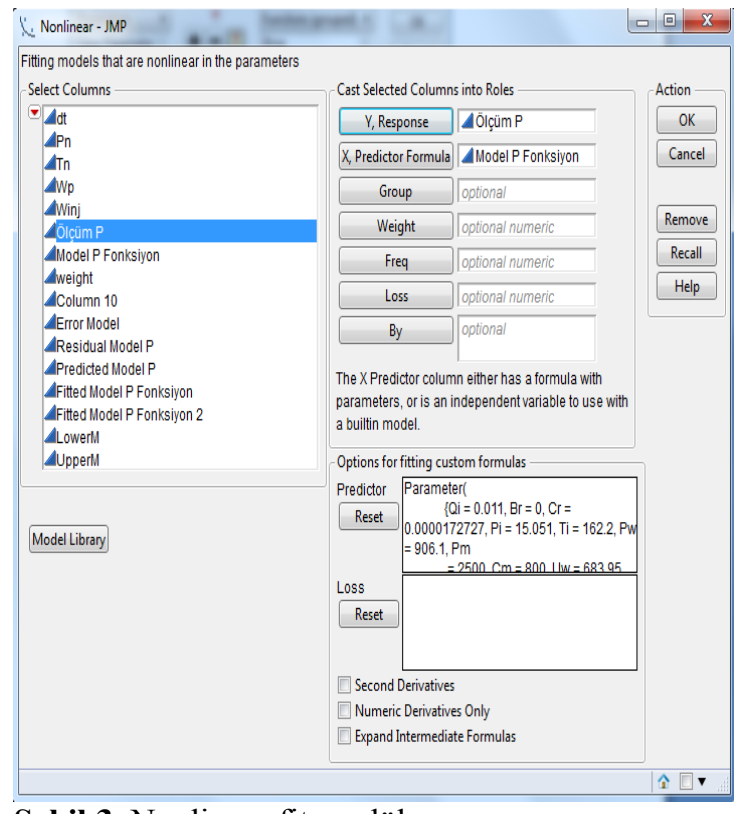

Şekil 3. Nonlineer fit modül

Model parametre değerlerinin kalibrasyonu sonucunda elde edilen parametre değerleri Çizelge 1'de karşılaştırmalı olarak verilmiştir.

Çizelge 1. Kalibre edilmiş model parametre değerleri

\begin{tabular}{|l|c|c|c|}
\hline \multicolumn{2}{|c|}{$\begin{array}{c}\text { Model Parametre } \\
\text { Değerleri }\end{array}$} & $\begin{array}{c}\text { Model İlk } \\
\text { Değerleri }\end{array}$ & $\begin{array}{c}\text { Gözlem Basıç } \\
\text { Değerleri ile Kalibre } \\
\text { Edilmiş Değerler }\end{array}$ \\
\hline $\mathrm{V}_{\mathrm{r}}$ & $\left(\mathrm{m}^{3}\right)$ & $1,39 \times 10^{9}$ & $1,39 \times 10^{9}$ \\
\hline$\rho_{\mathrm{m}}$ & $\mathrm{kg} / \mathrm{m}^{3}$ & 2.400 & 2.750 \\
\hline $\mathrm{Cm}$ & $\mathrm{J} /\left(\mathrm{kg}^{\circ} \mathrm{C}\right)$ & 800 & 1.000 \\
\hline $\mathrm{T}(\mathrm{i})$ & ${ }^{\circ} \mathrm{C}$ & 162,2 & 162,208432237576 \\
\hline $\mathrm{P}(\mathrm{i})$ & $\mathrm{bar}$ & 15,051 & 15,10 \\
\hline$\phi$ & $\%$ & 1,1 & 2,34756372992541 \\
\hline $\mathrm{P}_{\mathrm{w}}$ & $\mathrm{kg} / \mathrm{m}^{3}$ & 906,1 & 900,000000090808 \\
\hline $\mathrm{U}_{\mathrm{w}}$ & $\mathrm{kJ} / \mathrm{kg}$ & 683,95 & 683,95 \\
\hline $\mathrm{h}_{\mathrm{w}, \mathrm{s}}$ & $\mathrm{kJ} / \mathrm{kg}$ & 630 & 630,00 \\
\hline $\mathrm{h}_{\mathrm{w}, \mathrm{inj}}$ & $\mathrm{kJ} / \mathrm{kg}$ & 255,1 & 260,00 \\
\hline $\mathrm{h}_{\mathrm{w}, \mathrm{p}}$ & $\mathrm{kJ} / \mathrm{kg}$ & 685,65 & 685,65 \\
\hline $\mathrm{C}_{\mathrm{r}}$ & $1 / \mathrm{bar}$ & $1,7272 \times 10^{-5}$ & $1,32001 \times 10^{-5}$ \\
\hline$\beta$ & $1 /{ }^{\circ} \mathrm{C}$ & 0 & 0 \\
\hline$\alpha_{\mathrm{s}}$ & $\mathrm{m}^{3} / \mathrm{s}$ & 0,0645 & 0,619 \\
\hline
\end{tabular}




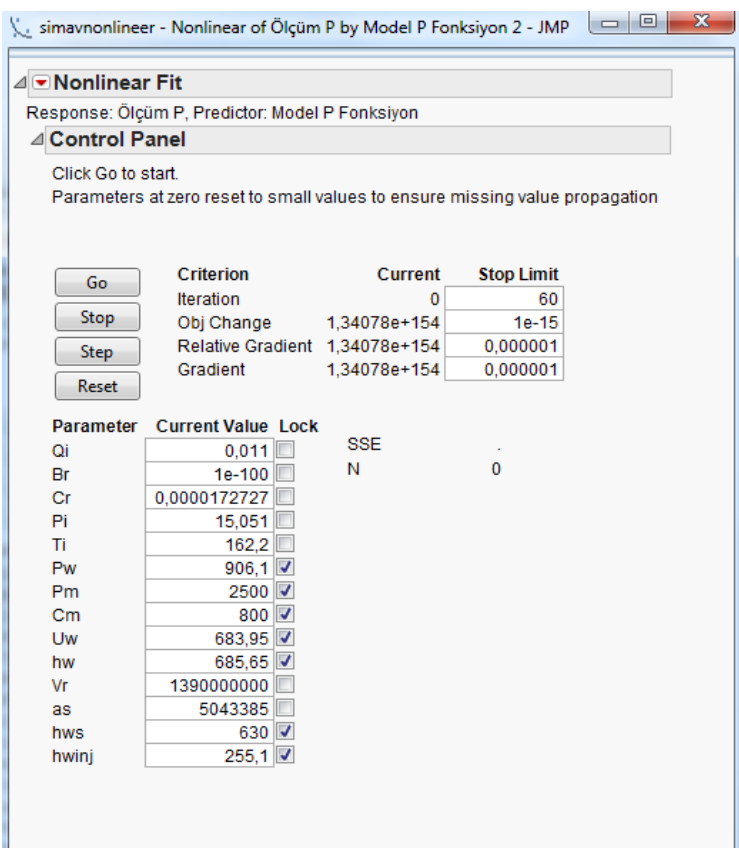

Şekil 4. Non-lineer regresyon modülü

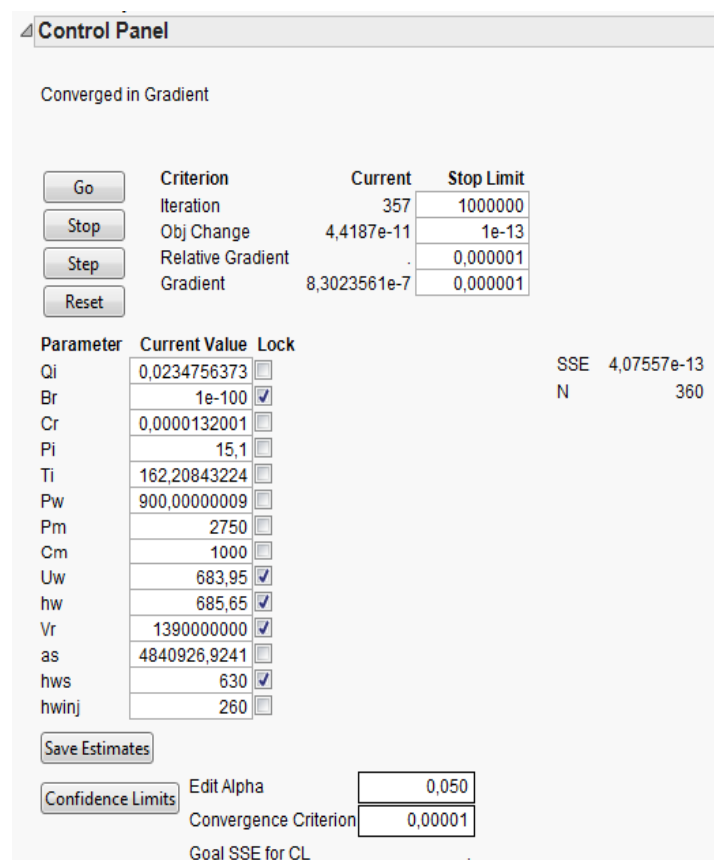

Şekil 5. Nonlineer regresyon sonucu

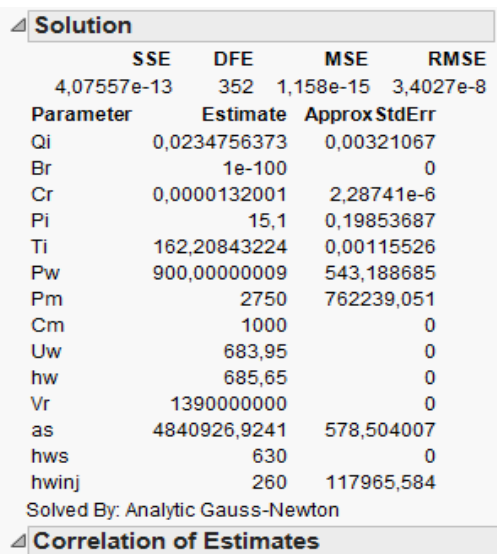

\begin{tabular}{|c|c|c|c|c|c|c|c|c|c|c|c|c|c|c|}
\hline & Qi & $\mathrm{Br}$ & $\mathrm{Cr}$ & $\mathrm{Pi}$ & $\mathrm{Ti}$ & Pw & $\mathrm{Pm}$ & $\mathrm{Cm}$ & Uw & $\mathrm{hw}$ & $\mathrm{Vr}$ & as & hws & hwinj \\
\hline Qi & 1,0000 & . & $-0,8655$ & $-0,9832$ & $-0,9999$ & $-0,9908$ & $-0,1554$ & & . & . & . & $-0,1397$ & . & 0,1546 \\
\hline $\mathrm{Br}$ & & . & & & & & & & . & . & . & & & \\
\hline $\mathrm{Cr}$ & $-0,8655$ & . & 1,0000 & 0,7637 & 0,8697 & 0,7900 & 0,5224 & & . & . & . & 0,5097 & & $-0,5217$ \\
\hline $\mathrm{Pi}$ & $-0,9832$ & & 0,7637 & 1,0000 & 0,9816 & 0,9976 & 0,0058 & & . & . & . & $-0,0100$ & & $-0,0050$ \\
\hline $\mathrm{Ti}$ & $-0,9999$ & . & 0,8697 & 0,9816 & 1,0000 & 0,9897 & 0,1659 & & . & . & . & 0,1502 & & $-0,1651$ \\
\hline $\mathrm{Pw}$ & $-0,9908$ & . & 0,7900 & 0,9976 & 0,9897 & 1,0000 & 0,0537 & & . & . & . & 0,0379 & & $-0,0529$ \\
\hline $\mathrm{Pm}$ & $-0,1554$ & . & 0,5224 & 0,0058 & 0,1659 & 0,0537 & 1,0000 & & . & . & . & 0,9999 & & $-1,0000$ \\
\hline $\mathrm{Cm}$ & & & . & . & . & & & & . & . & . & . & & \\
\hline Uw & & & . & . & . & & & & . & . & . & & & \\
\hline hw & & & . & . & . & & & & & . & . & & & \\
\hline $\mathrm{Vr}$ & & . & & & & & & & . & . & . & & & \\
\hline as & $-0,1397$ & & 0,5097 & $-0,0100$ & 0,1502 & 0,0379 & 0,9999 & & . & . & . & 1,0000 & & $-0,9999$ \\
\hline hws & & . & & & & & & & . & . & . & & & \\
\hline hwinj & 0,1546 & . & $-0,5217$ & $-0,0050$ & $-0,1651$ & $-0,0529$ & $-1,0000$ & & . & . & . & $-0,9999$ & & 1,0000 \\
\hline
\end{tabular}

Şekil 6. Nonlineer regresyon sonucu ve parametreler arası korelasyon 


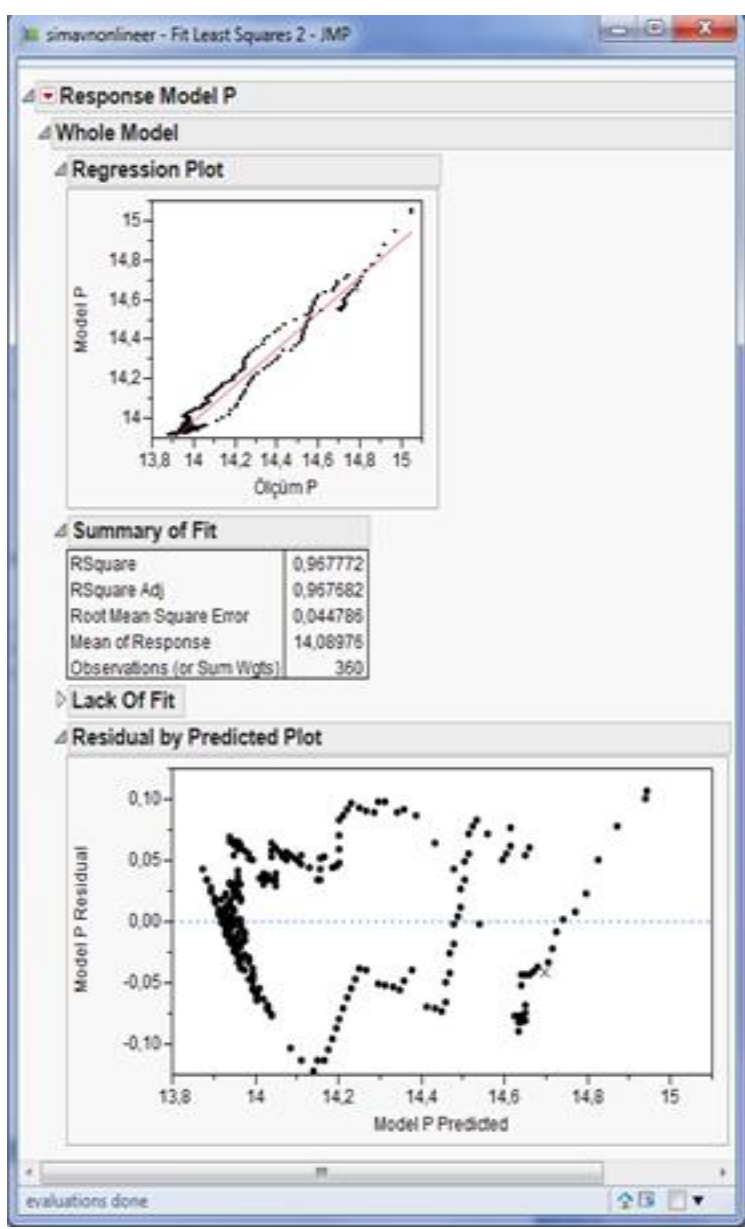

(a)

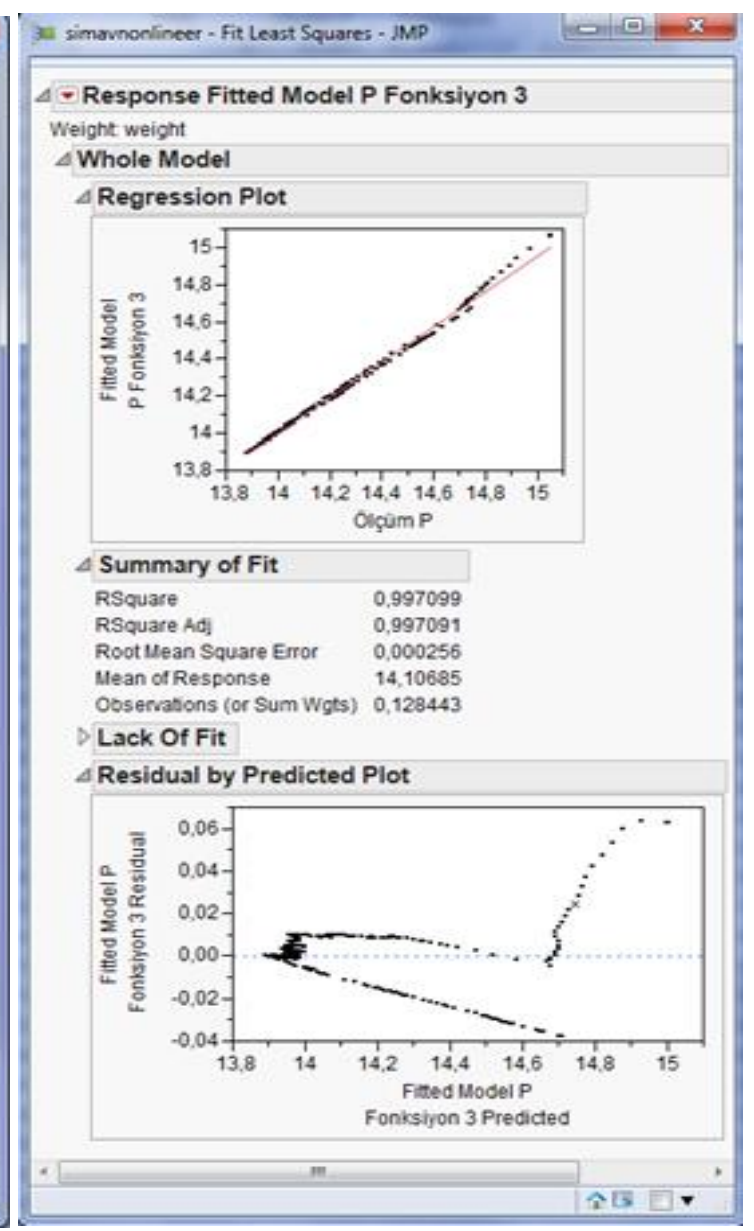

(b)

Şekil 7. Model parametre değerlerinin kalibresi öncesi (a) ve sonrası (b) fit model

Kalibre edilmiş parametre değerleri sonucunda model basınç değerleri ile gözlem basınç değerleri arasındaki ilişki incelenmiş olup, datalar arası ilişki \%99 seviyesine çıkmış, RMSE değeri ise 0,00084'den 0,00025 seviyesine düşmüştür (Şekil 7).

Model parametre değerlerinin doğrusal olmayan regrasyon ile kalibresi sonucu parametre değerleri ile residual değerleri model sistemine işlenmiş ve ölçüm/model basınç grafiği (Şekil 8) ile model sıcaklık grafiği (Şekil 9) çizilmiştir. Şekil 8'den de görüleceği gibi ölçüm/model basınç değerleri \%99 uyumludur. Model parametre değerlerinin kalibre edilmesi ve belirsizliklerin tespiti neticesinde, sahanın işletilmesinde saha basınç ve sıcaklık profilindeki değişimler tespit edilebilir ve irdelenebilir hale gelmiştir. $\mathrm{Bu}$ tespitler neticesinde, saha ile ilgili üretim-enjeksiyon optimizasyonu da gerçekleştirilebilir olmuştur. İşletmenin ihtiyaç duyduğu yıllık akışkan miktarı göz önünde bulundurularak, farklı senaryolar doğrultusunda saha model üzerinde işletilebilir ve sahada basınç kaybı en az olacak şekilde re-enjeksiyon miktarı tespit edilebilir olacaktır. 


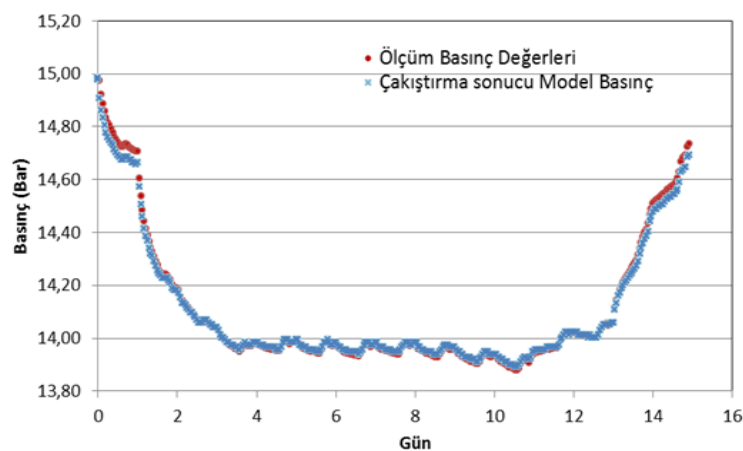

Şekil 8. İzotermal olmayan model basınç çakıştırma grafiği

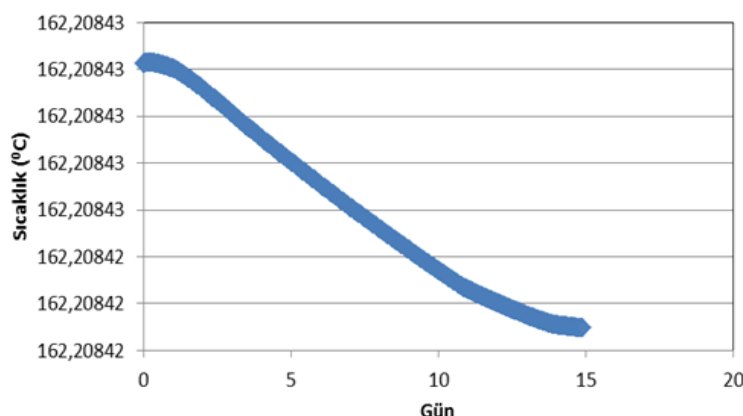

Şekil 9. İzotermal olmayan model sicaklık değişim profili

\section{SONUÇLAR}

İzotermal olmayan akış modeli, kütle korunum denklemi ile isı taşınım denkleminin birlikte çözülmesiyle elde edilir. Kütle ve enerji denklemleri doğrusal olmayan diferansiyel denklemler olduğundan tümüyle kapalı Newton-Raphson yineleme yöntemi ile birlikte çözümlenmelidir. Bu çözümlemeden sonra, saha parametrelerinin kalibrasyonu için sahadan gelen veriler, modelden elde edilen veriler ile çakıştırma yapilarak rezervuar parametreleri kalibre edilmelidir.

İzotermal olmayan akış modeli ile ölçüm verileri çakıştırması hem basınç, hem de sıcaklık verilerini birlikte kullanarak veya yalnızca basınç ve yalnızca sicaklık verileri ile yapılabilmektedir. Ancak, Kütahya-Simav sahasinda yapilan etkileşim test çalışmalarında, sadece kuyu dibi basınç verileri kaydedildiğinden çalışmada sadece basınç verilerine çakıştırma gerçekleştirilmiş ve çakıştırma neticesinde, tahmin edilen parametre değerleri kullanılarak sıcaklık profil değişimleri hesaplanmıştır.

Doğrusal olmayan model kalibarasyonu ile Pmodel ile Pölçüm değerleri uyumu \%96'dan \%99 seviyelerine ulaşmıştır. $\mathrm{R}^{2}$ değeri 0,044'den 0,00025 değerine düşmüştür. JMP Non-lineer model parametre tahmini modülü her çalıştırıldığında farklı değerlerle çözüm kümesi oluşturmaktadır. Parametrelerin kalibresi ile modülden model belirsizlikleri tespit edilir. Kalibre edilmiş model parametreleri ve belirsizlikler sisteme dahil edilerek modelleme sonlandirilır.

\section{KAYNAKLAR}

1. Whiting, R.L., Ramey, H.J., 1969. Application of Material and Energybalance to Geothermal Steam Production, Journal of Petroleum Technology, July, 893-900.

2. Bringham, W.E., Morrow, W.B., 1974. P/Z Behavior for Geothermal Steam Reservoirs. Paper SPE 4899 Presented at the $44^{\text {th }}$ Annual California Regional Meeting of the Society of Petroleum Engineers, AME, San. Francisco, California.

3. Bodvarsson, G.S., Pruess, K., Stefansson, V., Eliasson, E.T., 1984-b. The Krafla Geothermal Field, Iceland: 2. The Generating Capacity of the Field, Water Resources Research, 20 (11), 1531-1544.

4. Bodvarsson, G.S., Pruess, K., Lippman, M.J., 1986. Modeling of Geothermal Systems, Journal of Petroleum Technology, 1007-1021.

5. Alkan, H., Satman, A., 1990. A New Lumped Parameter Model for Geothermal Reservoirs in Presence of Carbon Dioxide, Geothermics, 19/5, 469-479.

6. Sarak, H., 2004. Düşük Sicaklıklı Jeotermal Rezervuarlar için Boyutsuz Rezervuar Modelleri, PhD Thesis, İTÜ, İstanbul, Türkiye.

7. Onur, M., Sarak, H., Türeyen, Ö.İ., Çınar, M., Satman, A., Korkmaz, E.D., 2008. Düşük Sicaklıklı Jeotermal Rezervuarların Akışkan ve Isı Üretim Davranışlarının Tank Modelleriyle Modellenmesi, Tübitak Projesi. 
8. Türeyen, Ö. İ., Onur, M., Sarak, H., 2009. A Generalized Nonisothermal Lumped-Parameter Model for Liquid Deominated Geothermal Reservoirs, $34^{\text {th }}$ Workshop on Geothermal Reservoir Engineering, Stanford University, Stanford, California, February 9-11.

9. Burden, R.L., Faires, J.D., 1989. Numerical Analysis, $4^{\text {th }}$ Edition, PWS-KENT Publishing Co., Boston.

10. Toraman, S., 2016. Kütahya-Simav Jeotermal Sahası Kuyu Etkileşimleri ve Üretim/Enjeksiyon Optimizasyonu, Çukurova Üniversitesi Fen Bilimleri Enstitüsü, Maden Mühendisliği Anabilim Dalı Doktora Tezi, 129, Adana. 
